\title{
Optical Aharonov-Bohm effect in stacked type-II quantum dots
}

\author{
Igor L. Kuskovsky, ${ }^{1, *}$ W. MacDonald, ${ }^{1}$ A. O. Govorov,${ }^{2}$ L. Mourokh,${ }^{1}$ X. Wei, ${ }^{3}$ M. C. Tamargo, ${ }^{4}$ \\ M. Tadic, ${ }^{5,6}$ and F. M. Peeters ${ }^{5}$ \\ ${ }^{1}$ Department of Physics, Queens College of CUNY, Flushing, New York 11367, USA \\ ${ }^{2}$ Department of Physics and Astronomy, Ohio University, Athens, Ohio 45701, USA \\ ${ }^{3}$ NHMFL, Tallahassee, Florida, 32310, USA \\ ${ }^{4}$ Department of Chemistry, The City College of CUNY, New York, New York 10031, USA \\ ${ }^{5}$ Department of Physics, University of Antwerp, Groenenborgerlaan 171, B-2020 Antwerp, Belgium \\ ${ }^{6}$ Faculty of Electrical Engineering, University of Belgrade, P.O. Box 3554, 11120 Belgrade, Serbia
}

(Received 15 February 2007; published 31 July 2007)

\begin{abstract}
Excitons in vertically stacked type-II quantum dots (QDs) experience a topological magnetic phase and demonstrate Aharonov-Bohm oscillations in emission intensity. The photoluminescence of vertically stacked $\mathrm{ZnTe} / \mathrm{ZnSe}$ QDs is measured in magnetic fields up to $31 \mathrm{~T}$. Aharonov-Bohm oscillations are found in the magnetic-field dependence of the emission intensity. The positions of the peaks of the emission intensity are in good agreement with numerical simulations of excitons in stacked QDs.
\end{abstract}

DOI: 10.1103/PhysRevB.76.035342

\section{INTRODUCTION}

The wave function of a quantum particle moving in a magnetic field will experience a phase shift proportional to the magnetic flux, $\Phi$, and the experimentally observable effects will be periodic with the period equal to the flux quantum, $\Phi_{0}=h / e$. This is the Aharonov-Bohm (AB) effect, ${ }^{1}$ which is typically observed as interference of a quantum charged particle moving along a closed trajectory in a magnetic field. Experimentally, many manifestations of the $\mathrm{AB}$ effect for charged particles have been observed. Optically, a signature of the $\mathrm{AB}$ effect has been reported for etched quantum rings ${ }^{2}$ where the $\mathrm{AB}$ effect is natural due to a nonzero total charge of a trion. Overall, the growth and magnetooptical characterization of epitaxial quantum rings are very active fields of research ${ }^{3-5}$ and the $A B$ effect is one of the central topics in these studies. Also, carbon nanotubes can demonstrate the $\mathrm{AB}$ features ${ }^{6}$ optically; however, small radii of nanotubes do not allow for observation the first $\mathrm{AB}$ oscillation. ${ }^{6}$

One of the most interesting problems in crystalline quantum rings is whether an overall neutral particle can exhibit the experimentally observable $\mathrm{AB}$ effect. ${ }^{7,8}$ Theoretical studies (see, e.g., Refs. 9-12) predict that such an effect indeed should be observed in the optical emission of neutral excitons in both quantum rings and type-II quantum dots (QDs). It is expected that the $A B$ phase will reveal itself through changes in both the excitonic energy and the intensity of the photoluminescence (PL). The latter can be quenched in a magnetic field that corresponds to the transition of the exciton angular momentum to a nonzero value. ${ }^{9-12} \mathrm{AB}$ oscillations of the excitonic energy with magnetic field have been reported for type-II InP/GaAs self-assembled QDs; ${ }^{13}$ however, the PL intensity dependence on the magnetic field has not yet been reported and is not well understood.

In this paper we present the results of experimental and theoretical studies of magnetoexcitons in type-II QDs formed in $\mathrm{Zn}-\mathrm{Se}-\mathrm{Te}$ multilayers that contain $\mathrm{ZnTe} / \mathrm{ZnSe}$ type-II QDs ${ }^{14}$ separated by relatively thin, nominally undoped, ZnSe
PACS number(s): 71.35.Ji, 78.55.Et, 78.67.Hc, 85.35.Be

barriers. Previously, the so-called optical AB effect for quantum rings and type-II QDs of various geometries was described in Refs. 9-12. These studies predict strong AB oscillations for exciton emission from quantum rings and type-II QDs if the exciton has a radial electric-dipole moment. We report here that the integrated PL intensity exhibits an oscillatory behavior as a function of magnetic field, as was predicted for a neutral exciton in type-II QDs. ${ }^{9-12}$ Theoretically, we explain the results as a motion of an electron around an entire stack of QDs, one of which is occupied by a hole. This suggests that a magnetic field can control optical emission of type-II nanostructures, which can be useful for quantuminformation-related applications.

\section{EXPERIMENTAL}

The type-II QDs studied in this work are formed in $\mathrm{Zn}$ Se-Te multilayers that are grown with submonolayer quantities of Te separated by nominally undoped ZnSe barriers. Although the growth procedure is described elsewhere (see, e.g., Ref. 15), we repeat some of the details here. Samples were grown by molecular beam epitaxy (MBE) on (001) GaAs substrates in a Riber 2300 system which includes III-V and II-VI growth chambers connected by an ultrahigh vacuum (UHV). Oxide desorption of the GaAs substrates was performed in the III-V chamber by heating the substrates to $590{ }^{\circ} \mathrm{C}$ with an As flux impinging on the surface. Then, a 200-nm GaAs buffer layer was grown at $580{ }^{\circ} \mathrm{C}$, and this gave a streaky $(2 \times 4)$ reflection high-energy electron diffraction (RHEED) surface pattern. The substrate with the GaAs buffer layer was transferred to the II-VI chamber under UHV. Prior to the growth of the II-VI epilayers, a Zn irradiation of the GaAs surface was performed. Then an undoped ZnSe buffer layer was grown at $250{ }^{\circ} \mathrm{C}$ under Se-rich conditions; RHEED gives a streaky $(2 \times 1)$ pattern. After the buffer layer growth, the multilayers were grown. Multiple monolayers (MLs) of a ZnSe spacer ( $>10$ MLs) were grown by opening the $\mathrm{Zn}$ and Se shutters together, after which the Se shutter was closed for $5 \mathrm{~s}$ to produce a $\mathrm{Zn}$-terminated 
surface. Then all shutters were closed for $5 \mathrm{~s}$ to desorb excess $\mathrm{Zn}$ from the surface. After this the Te shutter was opened for $5 \mathrm{~s}$ to deposit Te onto the Zn-terminated surface, and then all shutters were closed for $5 \mathrm{~s}$ followed by opening the $\mathrm{Zn}$ shutter for $5 \mathrm{~s}$ to produce another $\mathrm{Zn}$-terminated surface. It must be stressed that $\mathrm{Te}(\mathrm{Zn})$ is deposited without $\mathrm{Zn}$ (Te); such a growth procedure known as migration enhanced epitaxy (MEE), which enhances surface diffusion. Since a very small Te flux is used during the deposition, only a fraction of a Te monolayer (submonolayer) is formed (see Sec. III), which leads to formation of type-II QDs. This procedure was repeated three times, before the Se shutter was then opened to start the next growth sequence. This sequence was repeated for several periods in order to obtain layers thick enough for optical and structural measurement. The typical width of the period is $3 \mathrm{~nm}$; the tellurium composition within the barriers is less than $2 \%$, whereas the Te fraction in the QDs is typically $70 \%{ }^{16}$ In $\mathrm{ZnTe} / \mathrm{ZnSe}$ QDs the hole is strongly confined within the ZnTe-rich dot due to the larger valence band offset $(0.8-1.0 \mathrm{eV}),{ }^{17-19}$ whereas the electron is located within the $\mathrm{ZnSe}$-rich barriers.

Low-temperature $(T=4.2 \mathrm{~K})$ magnetophotoluminescence (magneto-PL) within the Faraday configuration up to $31 \mathrm{~T}$ on a series of $\mathrm{ZnTe} / \mathrm{ZnSe}$ multilayer QD samples was performed at the National High Magnetic Field Laboratory. For excitation either the 351-nm line of an $\mathrm{Ar}^{+}$laser or the $325-\mathrm{nm}$ line of a HeCd laser was used. The PL was detected by a liquid-nitrogen-cooled charge-coupled-device (CCD) camera coupled with a 1-m McPherson monocromator.

\section{MODEL AND CALCULATIONS}

The present system has important differences from the one studied in Ref. 13. First, the stacked cylindrical geometry nicely defines the ringlike trajectory for an electron, ensuring that the electron's wave function is "pushed" to the side of the dot, due to electron-electron interaction, independent of the stress in the system (see below). Indeed, our electron density calculations for a single dot show that the electron, in the absence of strain, will be located either above or below the dot (Fig. 1) and, therefore, no $\mathrm{AB}$ signature is expected. ${ }^{20}$ Second, a free particle here is the electron (rather than the hole as in Ref. 13), which has a smaller effective mass.

To calculate the exciton energies in stacks of $\mathrm{ZnTeSe} / \mathrm{ZnSe}$ QDs, we use a model proposed for type-II $\mathrm{InP} / \mathrm{InGaP}$ QDs and quantum-dot molecules ${ }^{10,21}$ and extend it here to a superlattice of disk-shaped QDs. The strain is taken to be constant inside the dot and zero in the spacer, which is a reasonable approximation for flat-shaped QDs. The relevant strain tensor components are extracted from the continuum mechanical model and are used in all dots. Because the spatial confinement and strain lift the energies of the heavy-hole states with respect to the light holes, only the heavy-hole exciton states are populated at $T=4.2 \mathrm{~K}$. The diagonal approximation to the multiband Hamiltonian is applied for the heavy hole and, therefore, different effective masses are used for the radial and vertical directions. ${ }^{22} \mathrm{We}$ further assume that the dot radii are different, but all dots have the same height and spacer thickness.

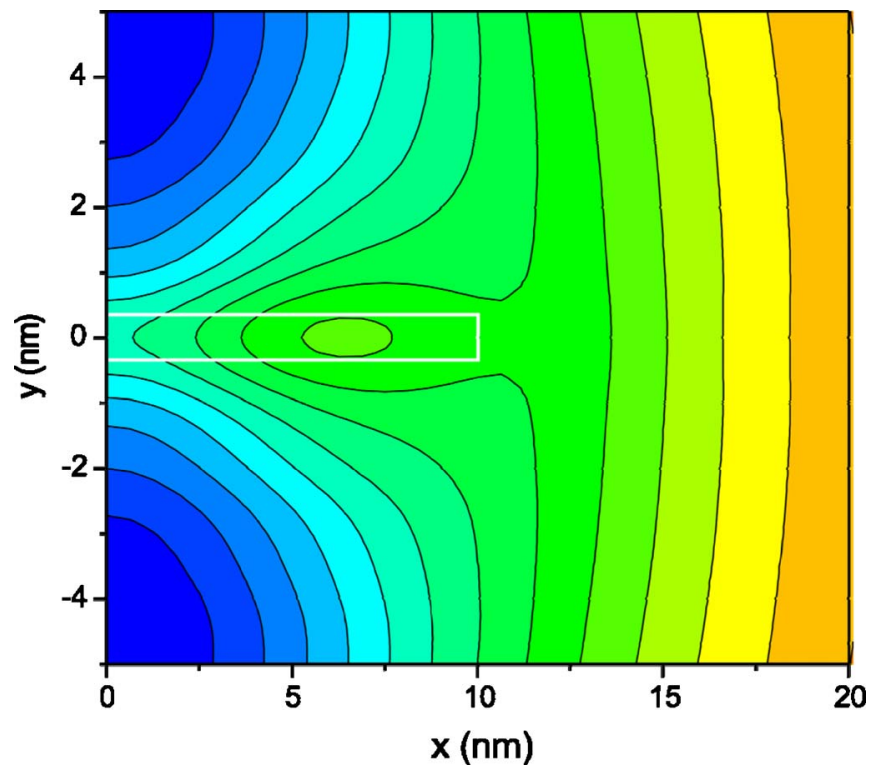

FIG. 1. (Color online) Electron charge density in the single dot. The dot boundary is shown by the white line. The electron is located above and below the dot. High (low) density is in blue (red) color.

Our model is shown schematically in Fig. 2(a). Numerical simulations show that tunneling of the hole is very weak and therefore the exciton should be trapped in one QD. To compute an exciton trapped in an infinite stack, we model a stack as a superlattice with a relatively long period. Each period in the stack consists of a single large dot of radius $R$ and seven smaller dots of radius $R_{1}=R-\Delta$ (for comparison with experiment, we took $\Delta=1 \mathrm{~nm}$ and $R=11 \mathrm{~nm}$ ). The exciton is trapped in the larger dot.

In order to find the single-particle states, the finiteelement method is employed to solve the Schrödinger equation. The exciton states are computed as follows. The Hartree iteration is used first to deliver a suitable basis for a subsequent exact-diagonalization procedure. The basis in the exact-diagonalization calculation is formed as the product of five electron wave functions of orbital momenta $l_{e}$ and six hole wave functions of orbital momenta $l_{h}$, such that $L=l_{e}$ $+l_{h}$ is the orbital momentum of the exciton, which is a conserved quantity and is fixed. In addition to the degeneracy with respect to $L$, the exciton states are arranged in quartets of spin-degenerate states in the absence of a magnetic field. Among the four states in the quartet only those with equal spins of the electron and the hole are optically active (see also below).

The dependence of the band gap in $\mathrm{ZnTe}_{x} \mathrm{Se}_{1-x}$ on $x$ is taken from Ref. 23. The experimental transition energy $\left(E_{\mathrm{exc}} \approx 2.46 \mathrm{eV}\right)$ is reproduced by selecting the conduction band offset of the strained structure $\Delta E_{C}=333 \mathrm{meV}$, while the valence band offset is $\Delta E_{V}=770 \mathrm{meV}$, in good agreement with previously reported unstrained values.${ }^{17-19}$ the first angular momentum transition is found at $B_{1} \approx 1.8 \mathrm{~T}$, when the radius of the large dot is taken to be $R=11 \mathrm{~nm}$ [Fig. 2(b)]. The thicknesses of both the dot, $h=0.7 \mathrm{~nm}$, which was chosen to be equal to the average thickness of the dot layer, and the spacer, $s=2.42 \mathrm{~nm}$, have been taken from the mea- 
(a)
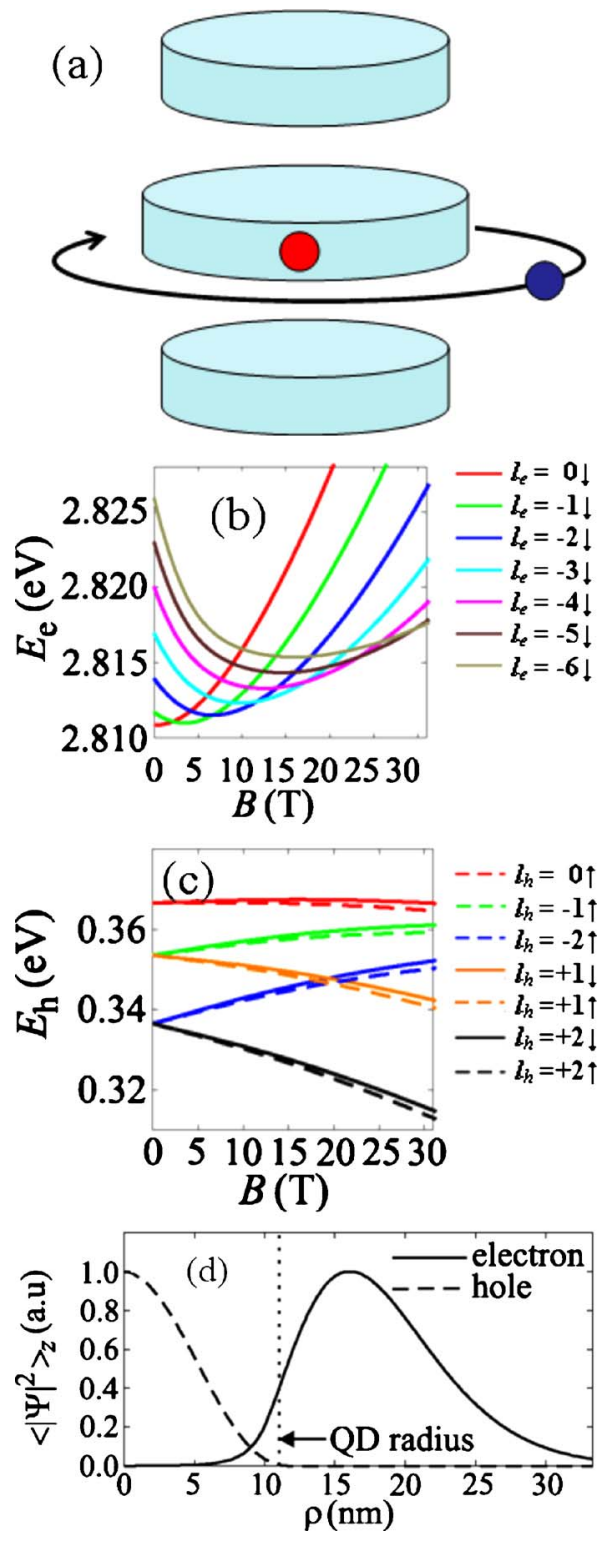

FIG. 2. (Color online) (a) Model of stacked type-II QDs with a hole localized within one dot and electrons free to move around the whole stack. (b) Dependence of the electron levels on magnetic field. (c) Dependence of the heavy-hole levels on magnetic field. (d) The probability densities of the ground electron and hole states averaged over $z, B=0 \mathrm{~T}$.

sured values. ${ }^{16}$ The parameters of the band structure and the permittivity in ZnTe and ZnSe are taken from Ref. 21, while the elastic constants and deformation potentials are taken from Ref. 24.

Figure 2(b) shows the variation of the lowest spin-down electron states of different orbital momenta with magnetic field. The hole is confined in a QD due to the built-in nanostructure potential while the electron is confined only due to the Coulomb interaction to the hole. The electron is distributed over a cylindrical surface, while the hole is strongly confined inside a QD. This spatial distribution of the particles determines the characters of the $B$ dispersion of the particle energies in the magnetic field. Figure 1(c) shows that, opposite to the electronic states, no angular momentum

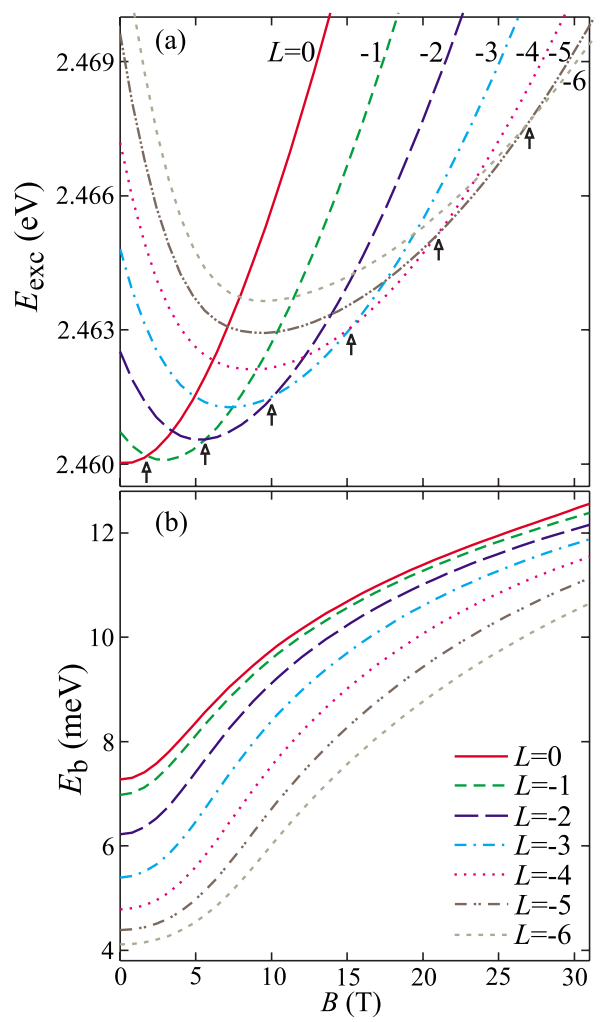

FIG. 3. (Color online) (a) Dependence of the computed spindown exciton energy levels on the magnetic field. (b) Binding energies of the lowest-energy exciton states of different orbital momenta.

transitions are found for the heavy-hole ground state. Both spin-up and spin-down hole states are shown [Fig. 2(c)]. The heavy-hole ground state has $l_{h}=0$ and the wave function is peaked in the center of the large dot, and its probability distribution is almost unaffected by the magnetic field. The smaller dots in the stack are not populated. Even though the chosen $\Delta=1 \mathrm{~nm}$ is only $10 \%$ of the radius of the large dot, the hole is localized in the large dot in each period. In order to illustrate the difference between the electron and hole localization in the structure, we plot in Fig. 2(d) the averages of the ground-state probability densities of the electron and hole ground states over the $z$ direction. These averages are scaled to span the same range.

The lowest-energy levels of spin-down exciton states are shown in Fig. 3(a) as functions of the magnetic field. The spin-up states have a similar variation, except that they have higher energies than the spin-down states. The states displayed in Fig. 3(a) exhibit several angular momentum transitions in the experimental range (see below) from 0 to $31 \mathrm{~T}$. In the axially symmetric structure, only the $L=l_{e}+l_{h}=0$ states are optically active, which would result in zero PL intensity beyond the first electron transition at $B_{1}=1.8 \mathrm{~T}$; note that the hole does not exhibit transitions. However, any deviation from perfect axial symmetry may turn the $L>0$ states optically active. ${ }^{25}$ For example, such deviations can appear for elongated QDs or in the presence of structural defects. Note that self-assembled QDs are typically elongated due to the specific symmetry of the sample surface. 


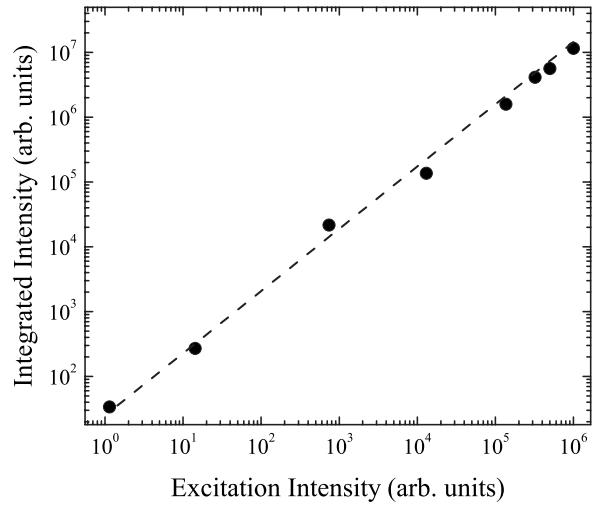

FIG. 4. Solid circles are measured integrated PL intensity $\left(I_{\mathrm{PL}}\right)$ as a function of excitation intensity $\left(I_{\text {exc }}\right)$. Dashed line is fitting to $I_{\mathrm{PL}} \sim I_{\mathrm{exc}}^{k}$ law $(k=0.96)$.

The computed binding energy of the exciton state at zero magnetic field equals $7.3 \mathrm{meV}$ [Fig. 3(b)], and as shown in Fig. 3(b), the binding energies of all states increase with magnetic field. The calculated dependence of the exciton energy levels shown in Fig. 3(a) is typical for quantum rings and type-II QDs. ${ }^{10}$ We note here that two subsequent angular momentum transitions at low magnetic field are separated by approximately $3.6 \mathrm{~T}$, while this difference increases to $6 \mathrm{~T}$ for $B \approx 27 \mathrm{~T}$. This is a clear sign that the effective exciton radius decreases with magnetic field. At low magnetic fields we find that $R_{\text {eff }}=19.1 \mathrm{~nm}$, and at high magnetic fields $(B$ $=27 \mathrm{~T}$ ) we come to $R_{e f f}=16.4 \mathrm{~nm}$.

\section{RESULTS AND DISCUSSION}

Figure 4 shows integrated PL intensity versus the excitation intensity at $B=0 \mathrm{~T}$. The data are fitted to an $I_{\mathrm{PL}} \sim I_{\text {exc }}^{k}$ law. ${ }^{26}$ The dependence is almost linear $(k \approx 0.96)$ over several orders of magnitude. This is possible if only relatively few QDs are occupied by holes, which supports our assumptions.

A typical magneto-PL from one of the samples (sample 1) for several values of the magnetic field and two excitation intensities is shown in Figs. 5(a) and 5(b). Furthermore, the spectra show the expected excitation-intensity-dependent spectral position ${ }^{14}$ at all magnetic fields. Figure 6 shows the data for three samples grown with increasing Te flux. ${ }^{27}$

The major feature of all samples is the presence of a strong oscillation in the integrated intensity. For the sample shown in Fig. 7 the peak is at $B=1.79 \pm 0.03 \mathrm{~T}$. The peak was masked at high excitation intensity [Fig. 7, inset (b)] by the emission due to isoelectronic bound excitons, ${ }^{14}$ which always exist in $\mathrm{Zn}-\mathrm{Se}-\mathrm{Te}$ systems. The observed behavior is reversible.

To understand the overall decrease in the PL intensity (e.g., Fig. 7), we point out that the system under study consists of multiple QD layers; thus, it is similar, in this respect, to multiple-quantum-well systems. In this case the interface defects are important, and they play the role of electron trapping centers, which results in a reduction of radiative recombination. Such a decrease in the overall PL intensity has been

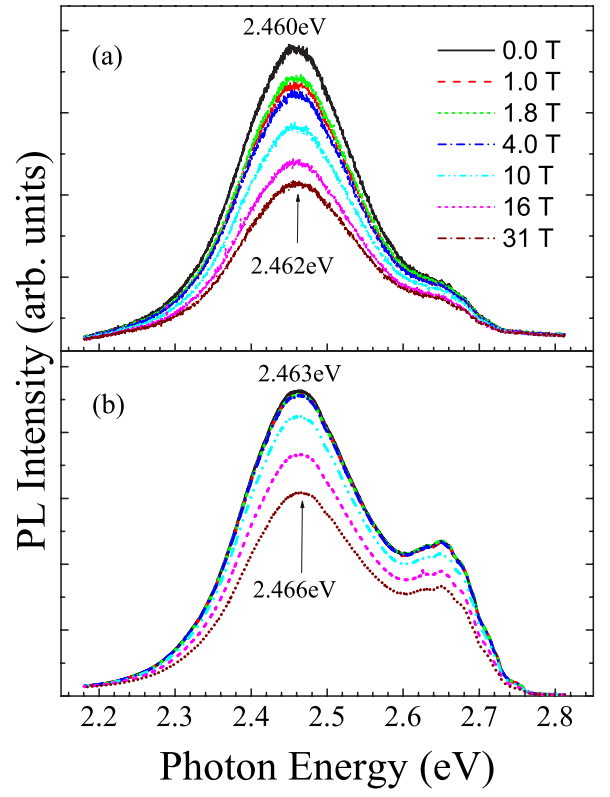

FIG. 5. (Color online) Magnetophotoluminescence from ZnTe/ZnSe multiple type-II QDs: (a) the lowest excitation intensity $I_{0}$; (b) the excitation intensity $100 I_{0}$.

indeed previously observed for type-II multiple-quantumwell structures. ${ }^{28,29}$ It is important to note that no oscillations in the PL intensity reported here were observed in Refs. 28 and 29; thus the presence of an interface or other defects cannot be viewed as contributing to the $\mathrm{AB}$ signatures observed here. On the other hand, it is consistent with the existence of cylindrical QDs as was suggested for this system earlier. ${ }^{14}$ Moreover, the overall decrease in PL intensity is also observed at high excitation [Fig. 7, inset b], where the $\mathrm{AB}$ signature is not seen, suggesting that this decrease is due to effects different from the $\mathrm{AB}$ one, and which are not re-

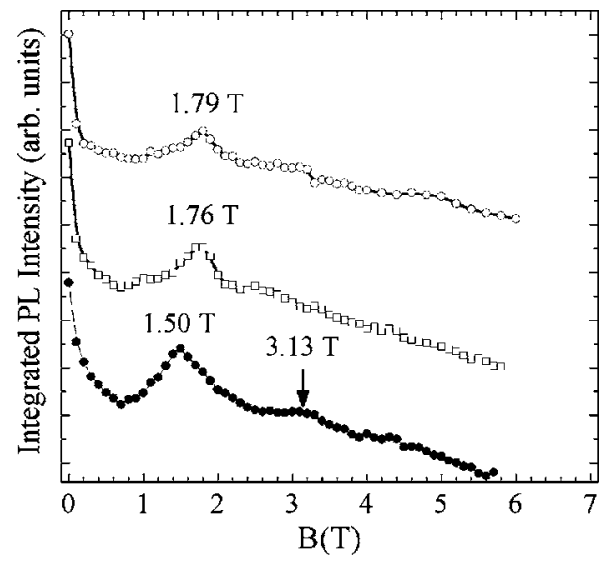

FIG. 6. Integrated magnetophotoluminescence from three $\mathrm{ZnTe} / \mathrm{ZnSe}$ type-II quantum-dot samples grown with increasing Te content (from top to bottom). The magnetic field of the angular momentum transition shifts to lower values. The first peak corresponds to a crossing point between $L=0$ and $L=-1$. The peak at 3.1 T observed in the lower curve is due to elongation of the QDs and is due to the crossing point between $L=0$ and $L=-2$ (see, e.g., Ref. 25). 


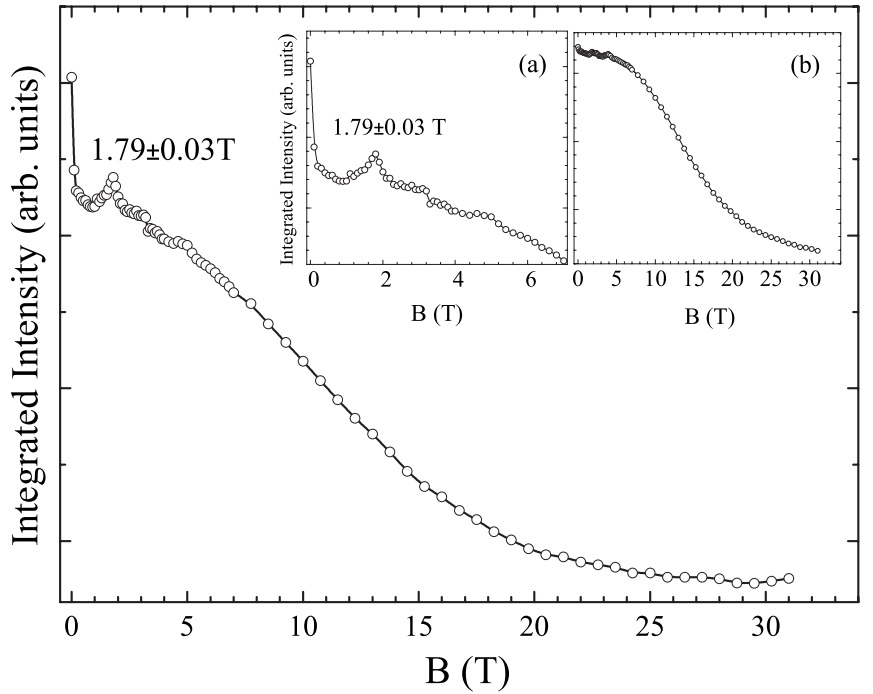

FIG. 7. Integrated magnetophotoluminescence from $\mathrm{ZnTe} / \mathrm{ZnSe}$ multiple type-II QDs at the lowest excitation intensity $I_{0}$. Inset (b) is the same for excitation $100 I_{0}$. Inset (a) shows a low-field region in greater detail.

sponsible for the observed oscillatory behavior. We thus argue that this peak is due to the $\mathrm{AB}$ phase as has been predicted earlier. The results of numerical calculations for this system as described above when compared with the experimental data provide reasonable values for the QD size. Furthermore, the use QD size $(R=11 \mathrm{~nm})$ in calculations of the binding energy is in excellent agreement with the measured 6-8 meV, ${ }^{14,30}$ which again supports our conclusions.

Moreover, results shown in Fig. 6 are consistent with the conclusions of $\mathrm{Gu}$ et al., ${ }^{30}$ who have shown that $\mathrm{Zn}-\mathrm{Se}-\mathrm{Te}$ multilayers grown with higher Te flux form larger QDs. Indeed, the magnetic field value required for the orbital momentum transition decreases with increasing Te concentration, indicating the presence of laterally larger QDs.

Next, we comment on the fact that PL does not vanish with increasing magnetic field and on the second feature observed at $B \approx 3.1 \mathrm{~T}$ in sample 3 . For ideal QDs with cylindrical symmetry, the theory predicts that PL transitions be- come forbidden at $B>B_{\text {crit }}$, where $\mathrm{B}_{\text {crit }}=\mathrm{B}_{1}$ is the magnetic field of the first ground-state transition for the electron $(L$ $=0 \rightarrow L=-1)$. Real QDs always have defects and are usually elongated. In the case of noncylindrical dots, the selection rules are relaxed and the dipole emission is allowed for an arbitrary $L,{ }^{25}$ so more features in the PL dependence on the magnetic field become possible. In particular, it was shown in Ref. 25 that the PL intensity does oscillate with magnetic field, especially at nonzero temperature, and the maxima in the $\mathrm{AB}$ oscillations correspond to the ground-state transitions in the exciton energy. The latter come from the fact that higher-energy exciton states contribute to the PL process. Our experiments demonstrate such oscillations; moreover, the flux values of the oscillations are in good agreement with the numerical data shown in Figs. 2(b) and 3(a). We assume that peaks in the magneto-PL intensity correspond to magnetic fields at which different exciton ground states cross each other in Fig. 3(a).

In conclusion, our model of the exciton states in $\mathrm{ZnTeSe} / \mathrm{ZnSe}$ QDs indicates that the wave function of the electron is localized around the radial periphery due to the joint action of the Coulomb interaction with the hole and the repulsive QD potential. Because of the smallness of the spacer layer between the QDs, the electron is unable to penetrate in the region between the dots, resulting in a favorable situation for the occurrence of the AB oscillations. Our magneto-PL data taken from several samples clearly show a nonmonotonous behavior of the exciton-emission intensity as a function of the magnetic field, which is a manifestation of the AB effect, and our numerical calculations support such an interpretation.

\section{ACKNOWLEDGMENTS}

This work was supported in part by DOE Award No. DEFG02-05ER46219, PSC-CUNY Award No. 68075-00 37, the EU-NoI: SANDiE, the Belgian Science Policy, and the Ministry of Science and Environmental Protection of the Republic of Serbia. A portion of this work was performed at the NHMFL, which is supported by NSF (Cooperation Agreement No. DMR-0084173) and by the State of Florida.
*Corresponding author. Igor.Kuskovsky@ qc.cuny.edu

${ }^{1}$ Y. Aharonov and D. Bohm, Phys. Rev. 115, 485 (1959).

${ }^{2}$ M. Bayer, M. Korkusinski, P. Hawrylak, T. Gutbrod, M. Michel, and A. Forchel, Phys. Rev. Lett. 90, 186801 (2003).

${ }^{3}$ P. Offermans, P. M. Koenraad, J. H. Wolter, D. Granados, J. M. Garcia, V. M. Fomin, V. N. Gladilin, and J. T. Devreese, Appl. Phys. Lett. 87, 131902 (2005).

${ }^{4}$ T. Kuroda, T. Mano, T. Ochiai, S. Sanguinetti, K. Sakoda, G. Kido, and N. Koguchi, Phys. Rev. B 72, 205301 (2005).

${ }^{5}$ B. Alen, J. Martinez-Pastor, D. Granados, and J. M. Garcia, Phys. Rev. B 72, 155331 (2005).

${ }^{6}$ S. Zaric, G. N. Ostojic, J. Kono, J. Shaver, and V. C. Moore, Science 304, 1129 (2004).
${ }^{7}$ A. V. Chaplik, JETP Lett. 62, 900 (1995).

${ }^{8}$ B. C. Lee, O. Voskoboynikov, and C. P. Lee, Physica E (Amsterdam) 24, 87 (2004).

${ }^{9}$ A. V. Kalameitsev, A. O. Govorov, and V. M. Kovalev, JETP Lett. 68, 669 (1998).

${ }^{10}$ K. L. Janssens, B. Partoens, and F. M. Peeters, Phys. Rev. B 64, 155324 (2001).

${ }^{11}$ A. O. Govorov, S. E. Ulloa, K. Karrai, and R. J. Warburton, Phys. Rev. B 66, 081309(R) (2002).

${ }^{12}$ J. I. Climente, J. Planelles, and W. Jaskólski, Phys. Rev. B 68, 075307 (2003).

${ }^{13}$ E. Ribeiro, A. O. Govorov, J. W. Carvalho, and G. MedeirosRibeiro, Phys. Rev. Lett. 92, 126402 (2004). 
${ }^{14}$ Y. Gu, I. L. Kuskovsky, M. van der Voort, G. F. Neumark, X. Zhou, and M. C. Tamargo, Phys. Rev. B 71, 045340 (2005).

${ }^{15}$ W. Lin, S. P. Guo, M. C. Tamargo, I. Kuskovsky, C. Tian, and G. F. Neumark, Appl. Phys. Lett. 76, 2205 (2000).

${ }^{16}$ The results are obtained from high-resolution symmetrical and asymmetrical high-resolution x-ray diffraction (HRXRD) and will be published elsewhere. Briefly, the symmetric (004) HRXRD curves in the $\omega-2 \theta$ mode show a spectrum typical of multilayer samples. Superlattice (SL) satellites up to second order are observed; all observed SL satellites are distributed with nearly equal angular separation, suggesting well-defined overall periodicity along the growth direction. The structural parameters were extracted via theoretical simulations of HRXRD curves, based on dynamical diffraction theory. There are two significant findings related to this work: the average QD-layer thickness corresponds to submonolayer quantities of Te expected from the growth conditions and a higher $\mathrm{Te} / \mathrm{Zn}$ flux ratio results in a higher average Te composition in the QD layers. The data on a similar system can be found in Y. Gong et al., J. Appl. Phys. 99, 064913 (2006).

${ }^{17}$ F. Malonga, D. Bertho, C. Jouanin, and J. M. Jancu, Phys. Rev. B 52, 5124 (1995).

${ }^{18}$ S.-H. Wei and A. Zunger, Phys. Rev. B 53, R10457 (1996).

${ }^{19}$ S.-H. Wei and A. Zunger, Appl. Phys. Lett. 72, 2011 (1998).

${ }^{20} \mathrm{We}$ note that the strain may tend to localize the electron at the edges of the QD, ${ }^{13}$ where the strain is maximal, and compete with the Coulomb-induced localization of electron on the top or bottom of the QD. The strain effects in single and stacked $\mathrm{ZnTe} / \mathrm{ZnSe}$ QDs need to be investigated more and magneticfield spectroscopy, which reveals the existence of ringlike trajectories via the $\mathrm{AB}$ effect, may be very useful.

${ }^{21}$ M. Tadic, F. M. Peeters, and K. L. Janssens, Phys. Rev. B 65, 165333 (2002).

${ }^{22}$ S. Lee, F. Michl, U. Rössler, M. Dobrowolska, and J. K. Furdyna, Phys. Rev. B 57, 9695 (1998).

${ }^{23}$ M. J. S. P. Brasil, R. E. Nahory, F. S. Turco-Sandroff, H. L. Gilchrist, and R. J. Martin, Appl. Phys. Lett. 58, 2509 (1991).

${ }^{24}$ J. Sörgel and U. Scherz, Eur. Phys. J. B 5, 45 (1998).

${ }^{25}$ L. G. G. V. Dias da Silva, S. E. Ulloa, and A. O. Govorov, Phys. Rev. B 70, 155318 (2004).

${ }^{26}$ T. Schmidt, K. Lischka, and W. Zulehner, Phys. Rev. B 45, 8989 (1992).

${ }^{27}$ The difference of $0.03 \mathrm{~T}$ between the uppermost and middle curves, although small, is real, as determined by detailed examinations of multiple runs.

${ }^{28}$ A. Trüby, M. Potemski, and R. Planel, Solid-State Electron. 40, 139 (1996).

${ }^{29}$ J. Haetty, M. Salib, A. Petrou, T. Schmiedel, M. Dutta, J. Pamulapati, P. G. Newman, and K. K. Bajaj, Phys. Rev. B 56, 12364 (1997).

${ }^{30}$ Y. Gu, Igor L. Kuskovsky, M. van der Voort, G. F. Neumark, X. Zhou, M. Munoz, and M. C. Tamargo, Phys. Status Solidi B 241, 515 (2004). 